Onkologe 2009 · 15:1063-1064

DOI 10.1007/s00761-009-1736-7

Online publiziert: 4. November 2009

(c) Springer Medizin Verlag 2009

\author{
K. Höffken \\ Universitätsklinikum Jena
}

\title{
Interdisziplinarität in der Onkologie - Nicht nach dem St. Florian-Prinzip!
}

anders aus. Sonst wäre es nicht notwendig, erneut in einem Schwerpunktheft dieses Thema zu beleuchten.

Kongressen der Fachgesellschaften, die sich mit der Onkologie beschäftigen, in Sonntagsreden und Fensterreden die Notwendigkeit beschworen, Krebskranke interdisziplinär zu betreuen und die Etablierung interdisziplinärer Strukturen und Prozesse (im Sinne von Tumorkliniken, gemeinsamen Tumorstationen, Tumorkonferenzen zur Diagnose- und Therapiefestlegung) nachdrücklich gefordert.

Während Interdisziplinarität in der Onkologie im angelsächsischen Sprachraum und im europäischen Ausland zum großen Teil vorbildhaft gelebt wird, hinkt diese, für eine optimale Versorgung von Krebskranken essenzielle Vorgehensweise in Deutschland erkennbar hinterher. Hauptschuldige sind Fächeregoismen, z. T. manifestiert in Weiterbildungsordnungen, die als Berufsausübungsrechte gedeutet werden. Dahinter steht eine Einstellung, die Voraussetzung für den Alleinvertretungsanspruch des jeweiligen Faches ist. Diese Einstellung gilt es zu überwinden.

Die moderne Medizin ist in ihrem Wissensstand bekanntlich an die Kapazitätsgrenzen eines Einzelnen gestoßen. Niemand kann heute mehr behaupten, einen kompletten Überblick über die Möglichkeiten und Grenzen der modernen Onkologie zu besitzen. Deshalb ist eine erkennbare Spezialisierung erfolgt, die ständig voranschreitet. Diese Spezialisierung verlangt Interdisziplinarität, um dem Patienten die bestmögliche Behandlung zukommen zu lassen. Die Realität sieht oft

\section{( Die gelebte Interdisziplina- rität ist von der geforderten immer noch weit entfernt}

In der Realität war - und ist immer noch zu einem erkennbaren Teil - die gelebte weit von der geforderten Interdisziplinarität entfernt. Vertreter der Zunft handelten nach dem St. Florian-Prinzip: Ja, aber die anderen sollen es machen.

Die Beiträge des vorliegenden Heftes zeigen, wie weit die deutsche Onkologie auf dem Weg zu einer gelebten Interdisziplinarität gekommen ist. Zunächst werden die Strukturen beleuchtet.

In der ambulanten Versorgung liegt, wie Schmitz und Heymanns darlegen, schon seit der Onkologievereinbarung von gischen Schwerpunktpraxen bei internistischen Onkologen/Hämatologen und den onkologisch verantwortlichen Fachärzten (Urologen, Gynäkologen u. a.), die in internistisch-onkologischen Arbeitskreise (Qualitätszirkeln) mit allen relevanten Disziplinen (Radiologie, Pathologie, Psychoonkologie etc.)zusammenarbeiten. Darüber hinaus kooperieren die Niedergelassenen transsektoral mit Pflegediensten einerseits und in Tumorkonferenzen der Kliniken andererseits.

Die stationäre Versorgung beginnt bei klinischen Tumorzentren, die sich häufig in regionalen Verbünden konstituie1994 die Verantwortung in den onkolo- ren. Hier sehen Zünkeler und Schlag eine wichtige Aufgabe, Schnittstellen zu optimieren und Schnittmengen zu definieren, um daraus professionelle Regionalverbünde mit Aufgaben in der ambulanten und stationären Versorgung zu schaffen. Auch hier wird die Forderung nach Interdisziplinarität über die Versorgungssektoren hinaus erkennbar.

Mit der Förderung onkologischer Spitzenzentren durch die Deutsche Krebshilfe wird jetzt die Interdisziplinarität solcher Einrichtungen in der Krankenversorgung festgeschrieben. Am Beispiel des Nationalen Centrums für Tumorerkrankungen (NCT) in Heidelberg beschreibt Wiestler Strukturen und Aufgaben dieser "Comprehensive Cancer Centers". Neben der interdisziplinären Krankenversorgung haben diese Zentren die Aufgabe, in der klinischen, kliniknahen und grundlagenwissenschaftlichen Forschung den Kenntnisstand zu erweitern und den zukünftig ebenfalls zu etablierenden klinisch-onkologischen Zentren zur Verfügung zu stellen. Letztere werden sich aus den existierenden regionalen Verbünden und/oder existierenden Organzentren bilden. $\mathrm{Ob}$ einzelnstehende Organzentren sinnvoll und nützlich sind, wird kontrovers gesehen.

Insgesamt befinden sich die Bemühungen um eine geschichtete Strukturierung der Onkologie in Deutschland jetzt auf dem richtigen Weg. Letztlich folgt sie Überlegungen der Arbeitsgemeinschaft Deutscher Tumorzentren von vor 20 Jahren. 
Medizinische Prozesse finden ihr Abbild in Leitlinien. Dabei wird a priori ein interdisziplinäres Vorgehen bei der Erstellung moderner $\left(\mathrm{S}_{3}\right.$-)Leitlinien angenommen.

Zwei Beiträge beschäftigen sich mit dieser wichtigen Thematik, einmal durch Weinbrenner für die behandelnden Ärzte, einmal - und dies wird zunehmend wichtiger - durch Sänger und Mitautoren für die Patienten. Gerade Patientenleitlinien "Übersetzungen" der medizinischen Leitlinien für medizinische Laien - müssen angesichts der Verbreitung elektronischer Medien sorgfältig erstellt und zertifiziert werden, um den informierten Patienten auch mit gesichertem Wissen auszustatten.

Welche Verbesserungen in Strukturen und Prozessen in der Versorgung von Krebskranken zu erreichen sind, wird am Beispiel der gastrointestinalen Tumoren durch Settmacher und Mitautoren beschrieben. Hier wird immanent durch die aufgezeigten Verbesserungen in der interdisziplinären Zusammenarbeit auch eine Verbesserung der Behandlungsergebnisse angenommen.

Letztlich steht aber die Verfügbarkeit von verlässlichen, oder besser gesagt von verwertbaren Ergebnissen der Versorgung noch im Schatten der erkennbar entwickelten und verbesserten Struktur- und Prozessqualität in der Onkologie. Dies liegt an der mangelnden Festlegung auf Messparameter und insbesondere an der mangelnden Erfassung solcher allgemein anerkannter Parameter durch klinische Krebsregister, letzteres durch den eklatanten Mangel an klinischen Krebsregistern und den Mangel an Bereitschaft der handelnden Personen, diese Instrumente zu nutzen, um Transparenz und Vergleichbarkeit zu ermöglichen. Der Beitrag von Hölzel und Mitautoren legt den Finger in diese offene Wunde. Auch wenn in differenzierter Weise die verfügbaren Ergebnisse dargelegt und viele Dogmen kritisch hinterfragt werden, bleibt die Notwendigkeit bestehen, auf der Basis verfügbarer (noch zu erhebender) Daten die Versorgungszukunft zu gestalten.

Wie soll dies funktionieren? Was ist zu tun?

Zum einen haben die Fachgesellschaften, allen voran die Deutsche Krebsgesellschaft, die Aufgabe, die vorhande- nen Aktivitäten zur Etablierung zukunftsorientierter Strukturen und Prozesse zu begleiten und zu unterstützen, damit die jetzt erkennbaren Schritte zu einer nachhaltigen Versorgungsstruktur führen. Die bisherigen Handlungsfelder sind, wie von Bruns dargestellt, interdisziplinär erstellte Leitlinien, interdisziplinäre Behandlungseinrichtungen und interdisziplinäre Arbeitsgruppen. Wichtig ist, dass die Fachgesellschaften handelnde Personen von der Notwendigkeit der Interdisziplinarität überzeugen und Hindernisse, insbesondere durch ordnungspolitische Vorgaben, beseitigen.

Zum anderen hat die Politik - besonders mit Blick auf die demographische Entwicklung mit der sich daraus ergebenden Zunahme der Krebserkrankungen - die Aufgabe, die jetzt eingeschlagenen Wege zu unterstützen und die z. T. modellhaft angestoßenen Maßnahmen auf eine gesicherte Basis zustellen, z. B. durch Übernahme in die Regelversorgung. Die Interdisziplinarität ist bei alldem Garant für eine optimale Versorgung des Tumorkranken.

Wie Weißbach und Schaefer detailliert darlegen, müssen statt finanzieller Anreize Reputationsvorteile für die Nachhaltigkeit der Modellvorhaben (integrierte Versorgung, medizinische Versorgungszentren etc.) sorgen. Ihr Plädoyer geht dahin, dass Politik gestaltet statt kontrolliert, die Fachgesellschaften ihre Aufgabe wahrnehmen, für Behandlungsstatt Gewinnoptimierung zu sorgen. Die Ärzteschaft muss sich auf ihre ureigensten Aufgaben besinnen: Jeder muss sein bestes Wissen und Können dem Kranken zur Verfügung stellen. Das ist gelebte Interdisziplinarität.

„Heiliger Sankt Florian, verschon' mein Haus, zünd“ andre an!“ ist auf einer Votivtafel an einer Hauswand zu lesen. Die Interdisziplinarität in der Onkologie ist auf dem Weg, sich von diesem jahrelang geübten Prinzip zu verabschieden - zum großen Nutzen des krebskranken Patienten.

\section{Horran}

\section{K. Höffken}

Für die Schwerpunktthemenherausgeber und die Herausgeber

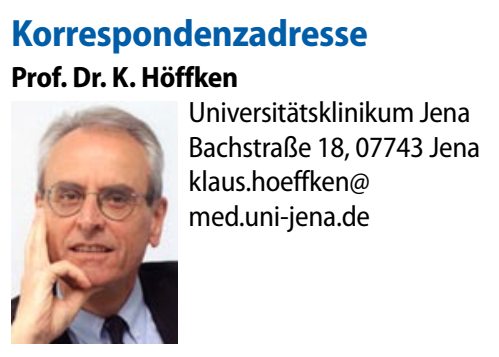

Interessenkonflikt. Der korrespondierende Autor gibt an, dass kein Interessenkonflikt besteht. 